\title{
VARIANTS OF UNCERTAIN TRANSSHIPMENT PROBLEM
}

\section{S. MOHANASELVI \& K. PRABAKARAN*}

Department of Mathematics, SRM Institute of Science and Technology, Kattankulathur, Tamilnadu, India

KEYWORDS: Uncertain Measure, Transshipment Problem, Impaired Flow, Enhanced Flow \& Chance Constrained Model

Received: May 13, 2020; Accepted: Jun 03, 2020; Published: Aug 03, 2020; Paper Id.: IJMPERDJUN2020577

\section{INTRODUCTION}

A transportation problem is a specialized optimization problem to lessen the transportation cost while transiting the trade of articles from different origins to destinations. But at some situations, intermediate nodes can be used to reach at the final destinations instead of transporting the goods directly from origins to destinations. Hence a transshipment problem can be used to ship the available articles to the respective destinations through some intermediate nodes at a reasonable cost. Alex Orden [1] introduced the classical transshipment problem in 1956 by ignoring the direct connections from origins to destinations and added a feature that shipment may go via any cycle of points. Later Hurt V.G. et al [2] formulated a multiregion, multiplant, multiprocessing, multiproduct transshipment problem and presented a solution procedure to it. The problem of minimizing the period of transportation problem with transshipment had been discussed by Rajeev Garg et al.[3]. Yale T. Herer et al [4] investigated the approach of transshipments in a dynamic deterministic demand environment. Khuranaetal $[5,6,7,8]$ had shown lights over three dimensional and variants of transshipment problem. Gregory Okiemute et al [9] presented a literature review on the emergence of the transshipment problem as well as the variants based on their objective functions. The parameters of transshipment problems are unpredictable in day today life due to various factors such as road condition, labor problem, incomplete information etc. To deal with the uncertainties present in the parameters different strategies such as probability theory, fuzzy set theory, evidence theory has been used. To assist the decision makers, researchers are still struggling to design the uncertainties present in decision making problems. Nagoorgani et al [10] had proposed an algorithm to solve mixed constraints transshipment problem in fuzzy environment. Rajendran et al [11] had obtained the optimal solution fora fully interval transshipment problem by using a split method. Hmiden. M et al [12] discussed about the fuzzy transshipment problem where the demands and the transfer lead time are represented by fuzzy numbers. 
Uncertainty theory is a innovative branch of mathematics for studying the performance of subjective uncertainty. Uncertainty theory was founded by Liu [13,18, 20] in 2007 for modeling belief degrees that human beings usually estimate. Uncertainty theory has been consequently explored by many researchers in literature. Yuhong Sheng et al [14] proposed a transportation problem based on uncertainty theory. Based on the uncertainty theory Bo Zhang et al [15] presented a hybrid intelligent algorithm design to solve fixed charge solid transportation problem. Subhakanta Dash et al [16] provided a solution procedure to transportation problem with uncertain cost, rough demand and supply. Yufu Ning et al [17] used triangular entropy of uncertain variable for a portfolio selection problem. A uncertain programming for transportation problem was discussed by DeyiMou [19].

In this paper an uncertain transshipment problem using uncertainty theory is addressed. All the parameters of the transshipment problems are treated as uncertain variables using its belief degree and solution procedure is presented in this paper. The rest of the paper is organized as follows: In section 2 preliminaries about uncertainty theory is introduced. In section 3 we introduce the uncertain transshipment model and its solution procedure. In section 4 an example is discussed to show the concepts developed in this paper. In section 5 conclusion of the paper is presented.

\section{PRELIMINARIES}

Generally in real life it is very difficult to predict a value of an event when it happens. The strength of the happening of an event is given by its belief degree. Liu [13] introduced uncertainty theory as an emerging tool to deal with belief degrees. The essential idea of uncertainty theory begins with uncertain measure. In this section we introduce various basic concepts of uncertainty theory.

\section{Definition 2.1[13]}

Let $\mathcal{L}$ "be a $\sigma$ - algebra defined over the non-empty universal set $\Gamma$. Every element $\Lambda \in \mathcal{L}$ is termed as an event. To express that chance an uncertain event to occur for any $\Lambda \in \mathcal{L}$ is given by an uncertain measure $\{\Lambda\}$. The set function $\mathcal{M}$ is called an uncertain measure if it satisfies the four axioms namely normality, duality, subadditivity and product axioms.

- Normality axiom: $\mathcal{M}^{\{\Gamma\}}=1$.

- Duality axiom: $\mathcal{M}\{\Lambda\}+\mathcal{M}\left\{\Lambda^{\mathrm{C}}\right\}=1$

- Subadditivity axiom: For every countable sequence of event $\Lambda_{1}, \Lambda_{2}, \ldots \ldots$ where

$$
\boldsymbol{M}\left\{\bigcup_{\mathrm{i}=1}^{\infty} \Lambda\right\} \leq \sum_{\mathrm{i}=1}^{\infty} \boldsymbol{\mathcal { C }}\left\{\Lambda_{\mathrm{i}}\right\}
$$

- Product axiom [Liu 2009]: The uncertainty space is defined by the triplet $(\Gamma, \mathcal{M})$. Let $\left(\Gamma_{\mathrm{k}}, \mathcal{L}_{\mathrm{k}}, \mathcal{M}_{\mathrm{k}}\right)$ be a uncertainty space for $\mathrm{k}=1,2, \ldots$, the product uncertain measure $\boldsymbol{M}$ satisfies

$$
\left\{\prod_{\mathrm{i}=1}^{\infty} \Lambda_{\mathrm{k}}\right\}=\bigwedge_{\mathrm{k}=1}^{\infty} \mathcal{M}_{\mathrm{k}}\left\{\Lambda_{\mathrm{k}}\right\}
$$


Definition 2.2[13]

$\xi:(\Gamma, \mathcal{L}, \mathcal{M}) \rightarrow \Re$ is an said to be an uncertain variable such that for any borel set $\mathrm{B}$ of real numbers $\{\xi \in \mathrm{B}\}$ is an event. That is

$$
\{\xi \in \mathrm{B}\}=\{\gamma \in \Gamma / \xi(\gamma) \in \mathrm{B}\}
$$

Definition 2.3[13]

If $\{\xi<0\}=0$, then the uncertain variable $\xi$ defined on $(\Gamma, \mathcal{L}, \mathcal{M})$ is said to be a non-negative uncertain variable and if $\boldsymbol{M}\{$ $\xi \leq 0\}=0$, then it said to be positive uncertain variable.

\section{Definition 2.4[13]}

For a uncertain variable $\xi$, the uncertainty distribution is defined by $\phi(x)=\{\xi \leq x\}$.

Note:1A uncertain variable $\xi$ is said to be linear if it has a linear uncertainty distribution $\phi(x)= \begin{cases}0 & \text { if } x \leq u \\ \frac{x-v}{v-u} & \text { if } u<x \leq v \\ 1 & \text { if } x>v\end{cases}$

and is denoted by $\xi \sim \mathcal{L}(\mathrm{u}, \mathrm{v})$ where uand $\mathrm{v}$ are real numbers with $\mathrm{u}<\mathrm{v}$.

Note: $2 \xi$ is said to be an zigzag uncertain variable if it has a uncertain distribution

$$
\phi(x)= \begin{cases}0 & \text { if } x \leq u \\ \frac{x-u}{2(v-u)} & \text { if } u<x \leq v \\ \frac{x+w-2 v}{2(w-v)} & \text { if } v<x \leq w \\ 1 \quad \text { if } x>w\end{cases}
$$

denoted by $\xi \sim \mathrm{Z}(\mathrm{u}, \mathrm{v}, \mathrm{w})$ where $\mathrm{u}$,vand $\mathrm{w}$ are real numbers with $\mathrm{u}<\mathrm{v}<\mathrm{w}$

Note: $3 \xi$ is said to be an normal uncertain variable if it has a normal uncertain distribution

$$
\phi(\mathrm{x})=\left(1+\exp \left(\frac{\pi(\mu-\mathrm{x})}{\sqrt{3} \sigma}\right)\right)^{-1}, \mathrm{x} \in \mathrm{R}
$$

denoted by $\xi \sim N(\mu, \sigma)$ where $\mu$ and $\sigma$ are real numbers with $\sigma>0$.

\section{Theorem 2.4:}

For uncertain variable $\xi$ with uncertain distribution $\phi(\mathrm{x})$ we have

$$
\mathcal{M}\{\xi \leq \mathrm{x}\}=\phi(\mathrm{x}) ; \mathcal{M}\{\xi>\mathrm{x}\}=1-\phi(\mathrm{x})
$$




\section{Definition 2.5:}

Let $\phi(\mathrm{x})$ be the regular uncertainty distribution of an uncertain variable $\xi$. Then the inverse uncertainty distribution of $\xi$ is given by the inverse function $\phi^{-1}(\alpha)$.

The inverse uncertainty distribution of $\mathrm{N}(\mu, \sigma)$ is defined as $\phi^{-1}(\alpha)=\mu+\frac{\sigma \sqrt{3}}{\pi} \ln \frac{\alpha}{1-\alpha}$

\section{Definition 2.6:}

Let $\xi$ be an uncertain variable with regular uncertain distribution $\phi(\mathrm{x})$. Then the expected value of $\xi$ is given"by $\mathrm{E}(\xi)=\int_{0}^{1} \phi^{-1}(\alpha) \mathrm{d} \alpha$

\section{Theorem 2.7}

Let $\xi$ be an uncertain variable with regular uncertain distribution function $\psi$.Then its $\alpha$-optimistic value and $\alpha-$ pessimistic values are $\xi_{\text {sup }}(\alpha)=\psi^{-1}(1-\alpha), \quad \xi_{\text {inf }}(\alpha)=\psi^{-1}(\alpha)$

\section{UNCERTAIN PROGRAMMING}

A mathematical programming problem whose objective function and constraints include uncertain variables is called uncertain programming problem. An uncertainty programming model was initiated by Liu in 2009. Let $\boldsymbol{x}$ be a decision vector and $\xi$ is an uncertain vector. Assume that $\xi=\left(\xi_{1}, \xi_{2}, \ldots ., \xi_{n}\right)$ in which $\xi_{1}, \xi_{2}, \ldots ., \xi_{n}$ are 'n' independent uncertain variables whose regular uncertainty distributions are given by $\phi_{1}, \phi_{2}, \ldots, \phi_{\mathrm{n}}$ respectively. An uncertain objective function $f(x, \xi)$ cannot be directly minimized and hence we may minimize the pessimistic value of the objective function with the predetermined value $\alpha \in(0,1)$.

$\min Z^{*}$

$$
\mathcal{M}\left(f(x, \xi) \leq Z^{*}\right) \geq \alpha
$$

The uncertain constraints $\boldsymbol{g}_{\mathrm{j}}(\boldsymbol{x}, \boldsymbol{\xi}) \leq 0 ; \mathrm{j}=1,2, \ldots \mathrm{p}$ do not define a deterministic feasible set and to get a desired solution the uncertain constraints are assumed to hold with confidence levels $\alpha_{1}, \alpha_{2}, \ldots, \alpha_{p}$ respectively. Then, the set of chance constraints is given by,

$$
\boldsymbol{M}\left(\mathbf{g}_{\mathrm{j}}(\mathrm{x}, \xi) \leq 0\right) \geq \alpha_{\mathrm{j}} ; \quad \mathrm{j}=1,2, \ldots \mathrm{p}
$$

Hence the chance constraint programming model of uncertain mathematical programming problem is given by

$$
\begin{aligned}
& \min Z^{*} \\
& \mathcal{M}\left(\mathbf{f}(\mathrm{x}, \xi) \leq \mathrm{Z}^{*}\right) \geq \alpha
\end{aligned}
$$


$\boldsymbol{M}\left(\mathbf{g}_{\mathrm{j}}(\mathrm{x}, \xi) \leq 0\right) \geq \alpha_{\mathrm{j}} ; \quad \mathrm{j}=1,2, \ldots \mathrm{p}$

We assume that $\boldsymbol{f}\left(\boldsymbol{x}, \xi_{1}, \xi_{2}, \ldots ., \xi_{\boldsymbol{n}}\right)$ is strictly increasing with respect to $\xi_{1}, \xi_{2}, \ldots ., \xi_{\mathrm{k}}$ and strictly decreasing with respect to $\xi_{\mathrm{k}+1}, \xi_{\mathrm{k}+2}, \ldots ., \xi_{\mathrm{n}}$ and similarly for the constraint function $\boldsymbol{g}_{\mathrm{j}}\left(\boldsymbol{x}, \xi_{1}, \xi_{2}, \ldots ., \xi_{\boldsymbol{n}}\right) \leq 0 ; \mathrm{j}=1,2, \ldots \mathrm{p}$ are strictly increasing with respect to $\xi_{1}, \xi_{2}, \ldots ., \xi_{\mathrm{k}}$ and strictly decreasing with respect to $\xi_{\mathrm{k}+1}, \xi_{\mathrm{k}+2}, \ldots . ., \xi_{\mathrm{n}}$ respectively. Then the equivalent deterministic model of (1) is given by

$\min Z^{*}$

subject to

$$
\begin{aligned}
& \boldsymbol{f}\left(\boldsymbol{x}, \phi_{1}^{-1}(\alpha), \phi_{2}^{-1}(\alpha), \ldots, \phi_{k}^{-1}(\alpha), \phi_{k+1}^{-1}(1-\alpha), \ldots, \phi_{n}^{-1}(1-\alpha)\right) \leq 0 \\
& \boldsymbol{g}_{j}\left(\boldsymbol{x}, \phi_{1}^{-1}(\alpha), \phi_{2}^{-1}(\alpha), \ldots, \phi_{k}^{-1}(\alpha), \phi_{k+1}^{-1}(1-\alpha), \ldots, \phi_{n}^{-1}(1-\alpha)\right) \leq 0 ; \quad j=1,2, \ldots p
\end{aligned}
$$

The deterministic programming model (2) can be solved by any existing methodand hence solution for uncertain programming model (1) is reached.

\section{MATHEMATICAL FORMULATION OF UNCERTAIN TRANSSHIPMENT PROBLEM}

Consider an uncertain transshipment problem with $\mathrm{m}$ sources and $\mathrm{n}$ destinations. In a uncertain transshipment problem a transshipment node can receive and sent product simultaneously from one node to another nodes. We define supply node as a node which only can sent to products but cannot receive them from any other nodes similarly a destination node can only receive the products but cannot sent the products to other nodes. Since in uncertain transshipment problem, each source and destination acts as transshipment nodes, for convenience the sources are labeled from 1 to $\mathrm{n}$ and destinations from $\mathrm{m}+1$ to $m+n$.

Let the uncertain availability at the sources be $\eta_{\mathbf{i}}(i=1,2, \ldots, \mathrm{m})$ and the uncertain requirement at the destinations be $\gamma_{\mathbf{j}}(\mathrm{j}=\mathrm{m}+1, \mathrm{~m}+2, \ldots, \mathrm{m}+\mathrm{n}, \mathrm{j} \neq \mathrm{i})$. The amount of products to be shipped from source $i$ to destination $j$ be $\boldsymbol{x}_{\mathrm{ij}}(\mathrm{i}, \mathrm{j}=1,2, \ldots, \mathrm{m}+\mathrm{n}, \mathrm{j} \neq \mathrm{i})$ .The unit uncertain cost of shipping the products from source $i$ to destination $j$ be $\xi_{\mathrm{ij}}(\mathrm{i}, \mathrm{j}=1,2, \ldots, \mathrm{m}+\mathrm{n}, \mathrm{j} \neq \mathrm{i})$ and $\xi_{\mathrm{ij}} \neq \xi_{\mathrm{ji}}$ (need not be same). Here $\phi_{\mathbf{i}}, \psi_{\mathbf{j}}, \phi_{\mathrm{ij}}$ are the uncertainty distributions of the independent uncertain variables $\eta_{\mathbf{i}}, \gamma_{\mathbf{j}}, \xi_{\mathrm{ij}}$ respectively. The number products to be transshipped through every transshipment node in or out cannot exceed the total number of products and let it be $S$ which can be treated as a buffer stock. That is $S=\max \left(\sum_{i=1}^{m} \eta_{i}, \sum_{j=m+1}^{m+n} \gamma_{j}\right)$.

Mathematically the uncertain transshipment problem is defined as follows 


$$
\begin{array}{lc}
\min \mathrm{f}(\boldsymbol{x}, \xi)=\sum_{i=1}^{m+n} \sum_{j=1}^{m+n} \xi_{i j} x_{i j} & (P 1) \\
\text { subject to } & \\
\sum_{j=1}^{m+n} x_{i j} \leq \eta_{i}+S, & i=1,2, \ldots, m \\
\sum_{j=1}^{m+n} x_{i j}=S, & i=m+1, \ldots, m+n \\
\sum_{i=1}^{m+n} x_{i j} \geq \gamma_{i}+S, & j=1,2, \ldots, n \\
\sum_{i=1}^{m+n} x_{i j}=S, & j=m+1, \ldots, m+n \\
x_{i j} \geq 0, & \forall i, j=1,2, \ldots, m+n, i \neq j \\
c_{i i}=0, \forall i,=1,2, \ldots, m+n . &
\end{array}
$$

\subsection{Uncertain Transshipment Problem with Impaired Flow}

The production factories may reserve some amount of products to handle emergency situations, thereby limiting the whole transshipment stream to a precise level R.

(i, e) $\sum_{i=1}^{m} \sum_{j=m+1}^{m+n} x_{i j}=R$ (or) $\sum_{i=1}^{m+n} \sum_{j=1}^{m+n} x_{i j}=R^{*}$ Where $R^{*}=R+(m+n) S$

Let the reserve stock kept at source be $\left(\sum_{i=1}^{m} \eta_{i}+(m+n) S\right)-R^{*}$ and the retained at various destinations be $\left(\sum_{j=m+1}^{m+n} \gamma_{j}+(m+n) S\right)-R^{*}$. Hence in model $P_{1}$ to receive the source stocks we have to introduce an additional destination and to fill up the destination stocks we have to introduce additional sources. The uncertain transshipment problem with restricted flow is as follows

$$
\begin{aligned}
& \min z=\sum_{\mathrm{i}=1}^{\mathrm{m}+\mathrm{n}+1} \sum_{\mathrm{j}=1, \mathrm{j} \neq \mathrm{i}}^{\mathrm{m}+\mathrm{n}+1} \xi_{\mathrm{ij}} \mathrm{y}_{\mathrm{ij}} \\
& \text { subject to } \\
& \sum_{\mathrm{j}=1}^{\mathrm{m}+\mathrm{n}+1} \mathrm{y}_{\mathrm{ij}} \leq \eta_{i}^{*}, \forall \mathrm{i}=1,2, \ldots, \mathrm{m}+\mathrm{n}+1 \\
& \sum_{\mathrm{i}=1}^{\mathrm{m}+\mathrm{n}+1} \mathrm{y}_{\mathrm{ij}} \geq \gamma_{j}^{*}, \forall \mathrm{j}=1,2, \ldots, \mathrm{m}+\mathrm{n}+1 \\
& \sum_{\mathrm{i}=1}^{\mathrm{m}+\mathrm{n}} \sum_{\mathrm{j}=1}^{\mathrm{m}+\mathrm{n}} \mathrm{x}_{\mathrm{ij}}=\mathrm{R}^{*} \quad\left(\mathrm{R}^{*}<\min \left\{\sum_{i=1}^{m} \eta_{\mathrm{i}}+(m+n) \mathrm{S}, \sum_{i=m+1}^{m+n} \gamma_{j}+(m+n) \mathrm{S}\right\}\right) \\
& \mathrm{y}_{\mathrm{ij}} \geq 0 ; i, j=1,2, \ldots, m+n+1 \\
& \text { where } \eta_{i}^{*}= \begin{cases}\eta_{i}+S & \forall i=1,2, \ldots, m \\
S & \forall i=m+1, m+2, \ldots, m+n \\
\sum_{j=1}^{m+n} \gamma_{j}^{*}-R^{*} \text { for } \mathrm{i}=\mathrm{m}+\mathrm{n}+1\end{cases}
\end{aligned}
$$




$$
\begin{aligned}
& \gamma_{j}^{*}= \begin{cases}\gamma_{j}+S & \forall j=m+1, m+2, \ldots, m+n \\
S & \forall j=1,2, \ldots, m \\
\sum_{i=1}^{m+n} \eta_{i}^{*}-R^{*} & \text { for } j=m+n+1\end{cases} \\
& \xi_{\mathrm{ij}}^{*}=\xi_{\mathrm{ij}} \quad \forall \mathrm{i}, \mathrm{j}=1,2, \ldots, \mathrm{m}+\mathrm{n} \\
& \xi_{\mathrm{i}, \mathrm{m}+\mathrm{n}+1}^{*}=\xi_{\mathrm{m}+\mathrm{n}+1, \mathrm{j}}^{*}=0, \forall \mathrm{i}, \mathrm{j}=1,2, \ldots, \mathrm{m}+\mathrm{n} \\
& \xi_{\mathrm{m}+\mathrm{n}+1, \mathrm{~m}+\mathrm{n}+1}^{*}=\mathrm{M} \quad \text { where is a large positive number }
\end{aligned}
$$

As we cannot obtain the deterministic feasible set directly for the uncertain transshipment problem, it is described to use chance constrained programming model. The pessimistic value of the objective function may be minimized with the predetermined value $\alpha, \alpha_{i}, \beta_{j} \in(0,1)$, so the uncertain transshipment problems P1, P2 can be reformed into the following model

$\min \mathrm{Z}^{*} \quad(\mathbf{P} 3)$

subject to

$\mathbf{M}\left\{\sum_{\mathrm{i}=1}^{\mathrm{m}+\mathrm{n}+1} \sum_{\mathrm{j}=1}^{\mathrm{m}+\mathrm{n}+1} \xi_{\mathrm{ij}}^{*} \mathrm{y}_{\mathrm{ij}} \leq \mathrm{Z}^{*}\right\} \geq \alpha$

Theorem 4.1: A feasible solution and optimal solution to P3 are the feasible and optimal solution to P2 respectively.Now we can transform P3 into the following equivalent model as follows:

$$
\begin{aligned}
\mathbf{M}\left\{\sum_{j=1}^{m+n+1} y_{i j}-\eta_{i}^{*} \leq 0\right\} \geq \alpha_{i} & \\
\text { ، }\left\{\left\{\gamma_{j}^{*}-\sum_{i=1}^{m+n+1} y_{i j}\right.\right. & \geq 0\} \geq \beta_{j} \\
\sum_{i=1}^{m+n} \sum_{j=1}^{m+n} y_{i j} & =R^{*} \\
y_{i j} & \geq 0 ; \quad i, j=1,2, \ldots, m+n+1
\end{aligned}
$$

$\min Z^{*}$

subject to

$$
\begin{gathered}
\sum_{\mathrm{i}=1}^{m+n+1} \sum_{j=1}^{m+n+1} \phi_{i j}^{-1}(\alpha) y_{i j}-Z^{*} \leq 0 \\
\sum_{j=1}^{m+n+1} y_{i j}-\phi_{i}^{-1}\left(1-\alpha_{i}\right)-S \leq 0 \quad \forall i=1,2, \ldots, m \\
\sum_{j=1}^{m+n} y_{i j}-S \leq 0 \quad \forall i=m+1, m+2, \ldots, m+n \\
\sum_{j=1}^{m+n} y_{i j}+\psi_{j}^{-1}\left(\beta_{j}\right)-R^{*} \leq 0 \quad i=m+n+1 \\
\psi_{j}^{-1}\left(\beta_{j}\right)-\sum_{i=1}^{m+n+1} y_{i j}+S \leq 0 \quad j=m+1, m+2, \ldots, m+n \\
\sum_{i=1}^{m+n} y_{i j}-S \leq 0 \quad \forall j=1,2, \ldots, m
\end{gathered}
$$


$\mathrm{P} 4$ is clearly a deterministic transformation problem satisfying the condition $\sum_{\mathbf{i}=1}^{m} \phi_{\mathbf{i}}^{-1}\left(1-\alpha_{\mathbf{i}}\right)=\sum_{\mathbf{j}=1}^{\mathbf{n}} \psi_{\mathbf{j}}^{-1}\left(\beta_{\mathbf{j}}\right)$ with confidence level $\alpha_{\mathbf{i}}, \beta_{\mathbf{j}} \in(0,1)$, then the following model P5 is called as balanced transportation problem.

$$
\begin{aligned}
& \min ^{\min } \quad(P 5) \\
& \sum_{\mathrm{i}=1}^{\text {subject to }} \sum_{\mathrm{j}=1}^{\mathrm{m}+\mathrm{n}+1} \phi_{\mathrm{ij}}^{\mathrm{m}+\mathrm{n}+1}(\alpha) \mathrm{y}_{\mathrm{ij}}-\mathrm{Z}^{*} \leq 0 \\
& \sum_{\mathrm{j}=1}^{\mathrm{m}+\mathrm{n}+1} \mathrm{y}_{\mathrm{ij}}-\phi_{\mathrm{i}}^{-1}\left(1-\alpha_{\mathrm{i}}\right)-\mathrm{S}=0 \quad \forall \mathrm{i}=1,2, \ldots, \mathrm{m} \\
& \sum_{\mathrm{j}=1}^{\mathrm{m}+\mathrm{n}} \mathrm{y}_{\mathrm{ij}}+\phi_{\mathrm{i}}^{-1}\left(1-\alpha_{\mathrm{i}}\right)-\mathrm{R}^{*}=0 \mathrm{i}=\mathrm{m}+\mathrm{n}+1 \\
& \psi_{\mathrm{j}}^{-1}\left(\beta_{\mathrm{j}}\right)+\mathrm{S}-\sum_{\mathrm{i}=1}^{\mathrm{m}+\mathrm{n}} \mathrm{y}_{\mathrm{ij}}-\mathrm{S}=0 \quad \forall \mathrm{i}=\mathrm{m}+1, \mathrm{~m}+2, \ldots, \mathrm{m}+\mathrm{n} \\
& \sum_{\mathrm{i}=1}^{\mathrm{m}+\mathrm{n}} \mathrm{y}_{\mathrm{ij}}-\mathrm{S}=0 \quad \forall \mathrm{j}=\mathrm{m}+1, \mathrm{~m}+2, \ldots, \mathrm{m}+\mathrm{n} \\
& \psi_{\mathrm{j}}^{-1}\left(\beta_{\mathrm{j}}\right)-\mathrm{R}^{*}-\sum_{\mathrm{i}=1}^{\mathrm{m}+\mathrm{n}} \mathrm{y}_{\mathrm{ij}}=0 \quad \mathrm{j}=\mathrm{m}+\mathrm{n}+1
\end{aligned}
$$

\subsection{Uncertain Transshipment Problem with Enhanced Flow}

The overall flow of the uncertain transshipment problem has to be enhanced due to need of additional demands in the market by increasing the production to meet up this additional demand. Let $R^{*}>\max \left(\sum_{\mathbf{i}=1}^{\mathbf{m}} \eta_{\mathbf{i}}+(m+n) T, \sum_{\mathbf{j}=\mathbf{m}+1}^{\mathbf{n}} \gamma_{\mathbf{j}}+(\mathrm{m}+\mathrm{n}) \mathrm{S}\right)$ be the enhanced flow.

A related transportation problem is formulated by adding an extra row with $R^{*}-\left(\sum_{i=1}^{m} \eta_{i}+(m+n) S\right)$ and an extra column with $\mathrm{R}^{*}-\left(\sum_{\mathbf{i}=1}^{\mathrm{m}} \gamma_{\mathrm{j}}+(\mathrm{m}+\mathrm{n}) \mathrm{S}\right)$ to manage the enhanced flow introduced due to transshipment. The uncertain transshipment problem with enhanced flow is given by

$$
\begin{aligned}
& \min \mathrm{z}=\sum_{i=1}^{m+n+1} \sum_{j=1, j \neq i}^{m+n+1} \xi_{i j} y_{i j} \quad(P 6) \\
& \text { subject to } \\
& \sum_{j=1}^{m+n+1} y_{i j} \leq \eta_{\mathrm{i}}^{*}, \forall i=1,2, \ldots, m+n+1 \\
& \sum_{i=1}^{m+n+1} y_{i j} \geq \gamma_{\mathrm{j}}^{*}, \forall j=1,2, \ldots, m+n+1 \\
& \sum_{i=1}^{m+n} \sum_{j=1}^{m+n} x_{i j}=R^{*} \quad R^{*}>\max \left(\sum_{\mathrm{i}=1}^{\mathrm{m}} \eta_{\mathrm{i}}+(m+n) S, \sum_{\mathrm{j}=\mathrm{m}+1}^{\mathrm{n}} \gamma_{\mathrm{j}}+(m+n) S\right) \\
& \text { ، } y_{i j} \geq 0 ; \mathrm{i}, \mathrm{j}=1,2, \ldots, \mathrm{m}+\mathrm{n}+1
\end{aligned}
$$




$$
\begin{aligned}
& \eta_{\mathrm{i}}^{*}= \begin{cases}\eta_{\mathrm{i}}+\mathrm{S} & \forall \mathrm{i}=1,2, \ldots, \mathrm{m} \\
\mathrm{S} & \forall \mathrm{i}=\mathrm{m}+1, \mathrm{~m}+2, \ldots, \mathrm{m}+\mathrm{n} \\
\mathrm{R}^{*}-\sum_{\mathrm{j}=1}^{\mathrm{m}+\mathrm{n}} \eta_{\mathrm{i}}^{*} & \text { for } \mathrm{i}=\mathrm{m}+\mathrm{n}+1\end{cases} \\
& \gamma_{\mathrm{j}}^{*}= \begin{cases}\gamma_{\mathrm{j}}+\mathrm{S} & \forall \mathrm{j}=\mathrm{m}+1, \mathrm{~m}+2, \ldots, \mathrm{m}+\mathrm{n} \\
\mathrm{S} & \forall \mathrm{j}=1,2, \ldots, \mathrm{m} \\
\mathrm{R}^{*}-\sum_{\mathrm{i}=1}^{\mathrm{m}+\mathrm{n}} \gamma_{\mathrm{j}}^{*} \text { for } \mathrm{j}=\mathrm{m}+\mathrm{n}+1\end{cases} \\
& \xi_{\mathrm{ij}}^{*}=\xi_{\mathrm{ij}} \quad \forall \mathrm{i}, \mathrm{j}=1,2, \ldots, \mathrm{m}+\mathrm{n} \\
& \xi_{\mathrm{i}, \mathrm{m}+\mathrm{n}+1}^{*}= \begin{cases}\min \sum_{\mathbf{j}=\mathbf{m}+1}^{\mathbf{m}+\mathbf{n}}\left\{\xi_{\mathrm{ij}}\right\} & \forall \mathrm{i}=1,2, \ldots, \mathrm{m} \\
\min \sum_{\mathbf{j}=1}^{\mathbf{m}+\mathbf{n}}\left\{\xi_{\mathrm{ij}}\right\} & \forall \mathrm{i}=\mathrm{m}+1, \mathrm{~m}+2, \ldots, \mathrm{m}+\mathrm{n}\end{cases} \\
& \xi_{\mathrm{m}+\mathrm{n}+1, \mathrm{j}}^{*}= \begin{cases}\min \sum_{\mathbf{j}=\mathbf{m}+1}^{\mathbf{m}+\mathbf{n}}\left\{\xi_{\mathrm{ij}}\right\} & \forall \mathrm{i}=1,2, \ldots, \mathrm{m} \\
\min \sum_{\mathbf{j}=1}^{\mathbf{m}+\mathbf{n}}\left\{\xi_{\mathrm{ij}}\right\} & \forall \mathrm{i}=\mathrm{m}+1, \mathrm{~m}+2, \ldots, \mathrm{m}+\mathrm{n}\end{cases} \\
& \xi_{\mathrm{m}+\mathrm{n}+1, \mathrm{~m}+\mathrm{n}+1}^{*}=\mathrm{M} \quad \text { where Mis a large positive number } \\
& \mathrm{y}_{\mathrm{ij}} \geq 0 \forall \mathrm{i}, \mathrm{j}=1,2, \ldots, \mathrm{m}+\mathrm{n}+1
\end{aligned}
$$

As we cannot obtain the deterministic feasible set directly for the uncertain transshipment problem, it is described to use chance constrained programming model. The pessimistic value of the objective function may be minimized with the predetermined value $\alpha, \alpha_{i}, \beta_{j} \in(0,1)$, and hence the uncertain transshipment problems P6 can be reformed into the following model

$\min Z^{*}$

subject to

$$
\begin{gathered}
\sum_{i=1}^{m+n+1} \sum_{j=1}^{m+n+1} \phi_{i j}^{-1}(\alpha) y_{i j}-Z^{*} \leq 0 \\
\sum_{j=1}^{m+n+1} y_{i j}-\phi_{i}^{-1}\left(1-\alpha_{i}\right)-S=0 \quad \forall i=1,2, \ldots, m \\
\sum_{j=1}^{m+n} y_{i j}-S=0 \quad \forall i=m+1, m+2, \ldots, m+n \\
\sum_{j=1}^{m+n} y_{i j}+\phi_{i}^{-1}\left(1-\alpha_{i}\right)-R^{*}=0 i=m+n+1 \\
\psi_{j}^{-1}\left(\beta_{j}\right)+S-\sum_{i=1}^{m+n+1} y_{i j}=0 \quad j=m+1, m+2, \ldots, m+n \\
\sum_{i=1}^{m+n} y_{i j}-S=0 \quad \forall j=1,2, \ldots, m \\
\psi_{j}^{-1}\left(\beta_{j}\right)-R^{*}-\sum_{i=1}^{m+n} y_{i j}=0 \quad j=m+n+1
\end{gathered}
$$

P7 is clearly a deterministic transformation problem and if P7 satisfy the condition $\sum_{\mathbf{i}=1}^{\mathrm{m}} \phi_{\mathbf{i}}^{-1}\left(1-\alpha_{\mathbf{i}}\right)=\sum_{\mathbf{j}=1}^{\mathrm{n}} \psi_{\mathbf{j}}^{-1}\left(\beta_{\mathbf{j}}\right)$ with 
confidence level $\alpha_{i}, \beta_{j} \in(0,1)$, then it is called as balanced transshipment problem.

\section{ILLUSTRATIVE EXAMPLES}

In this section we discuss an example of uncertain transshipment problem with impaired and enhanced flow.

\subsection{Illustrative Example for Uncertain Transshipment Problem with Impaired Flow}

The following uncertain transshipment problem with two sources and two destinations is considered.

The uncertain availabilities $\left(\eta_{\mathbf{i}}\right)$, uncertain requirements $\left(\gamma_{\mathbf{j}}\right)$ and uncertain $\operatorname{cost}\left(\xi_{\mathrm{ij}}\right)$ are represented as follows

$$
\begin{aligned}
& \xi_{i j} \sim N\left(\mu_{i j}, \sigma_{i j}\right) \quad i, j=1,2, \ldots j \neq i \\
& \eta_{i} \sim N\left(\mu_{i}, \sigma_{i}\right) \quad i=1,2, \ldots \\
& \gamma_{j} \sim N\left(\mu_{j}, \sigma_{j}\right) \quad j=1,2, . .
\end{aligned}
$$

Table 5.1: Uncertain Cost Matrix of Transshipment Problem

\begin{tabular}{|c|c|c|c|c|c|}
\hline $\mathbf{j}$ & $\mathbf{S 1}$ & $\mathbf{S 2}$ & $\mathbf{D 1}$ & $\mathbf{D} 2$ & $\eta_{\mathbf{i}}$ \\
\hline S1 & 0 & $\mathrm{~N}(1,1)$ & $\mathrm{N}(5,2)$ & $\mathrm{N}(3,1.5)$ & $\mathrm{N}(8,2)$ \\
\hline S2 & $\mathrm{N}(1,1)$ & 0 & $\mathrm{~N}(2,1)$ & $\mathrm{N}(4,2)$ & $\mathrm{N}(9,2)$ \\
\hline D1 & $\mathrm{N}(5,2)$ & $\mathrm{N}(2,1)$ & 0 & $\mathrm{~N}(2,1)$ & - \\
\hline $\mathrm{D} 2$ & $\mathrm{~N}(3,1.5)$ & $\mathrm{N}(4,2)$ & $\mathrm{N}(2,1)$ & 0 & - \\
\hline$\gamma_{\mathbf{j}}$ & - & - & $\mathrm{N}(3,1.5)$ & $\mathrm{N}(6,4)$ & \\
\hline
\end{tabular}

Table 5.2: Deterministic Cost Matrix for Predetermined Values $\alpha, \alpha_{i}, \beta_{j}=0.9(i, j=1,2,3,4)$

\begin{tabular}{|c|c|c|c|c|c|}
\hline $\mathbf{j}$ & $\mathbf{S 1}$ & $\mathbf{S 2}$ & $\mathbf{D 1}$ & $\mathbf{D 2}$ & $\phi_{\mathbf{i}}^{-1}(1-\alpha)$ \\
\hline $\mathbf{i} 1$ & 0 & 2.211 & 7.423 & 4.817 & 21.24 \\
\hline S2 & 2.211 & 0 & 3.211 & 6.423 & 22.24 \\
\hline D1 & 7.423 & 3.211 & 0 & 3.211 & 15.663 \\
\hline D2 & 4.817 & 6.423 & 3.211 & 0 & 15.663 \\
\hline D3 & 0 & 0 & 0 & 0 & 3.509 \\
\hline$\psi_{\mathbf{j}}^{-1}(\beta)$ & 15.663 & 15.663 & 20.48 & 26.509 & \\
\hline
\end{tabular}

By taking the predetermined confidence level as $\alpha=0.9, \alpha_{i}=0.9(i=1,2,3,4)$ and $\beta_{j}=0.9(j=1,2,3,4)$ Table 5.2 is formulated as table 5.3.

Table 5.2 is formulated by taking the predetermined confidence level as $\alpha=0.9, \alpha_{i}=0.9(i=1,2,3,4)$ and $\beta_{j}=0.9(j=1,2,3,4) \quad$ Since $\quad \sum_{j=3}^{4} \psi_{j}^{-1}(\beta)>\sum_{i=1}^{2} \phi_{i}^{-1}(1-\alpha) \quad$ we $\quad$ have $\mathrm{S}=\operatorname{Max}\left(\sum_{\mathrm{j}=3}^{4} \psi_{\mathbf{j}}^{-1}(\beta)>\sum_{\mathbf{i}=1}^{2} \phi_{\mathbf{i}}^{-1}(1-\alpha)\right)=15.663$. Let $\mathrm{R}^{*}$ be the total flow limited to 70.903 . We introduce a additional row and column with unit cost 0 except $\mathrm{C}_{55}$ having cost $\mathrm{M}$.

The dummy column O3 is allotted a demand $=\sum_{\mathbf{i}=1}^{4} \phi_{\mathbf{i}}^{-1}(1-\alpha)-\mathrm{R}^{*}=74.806-70.903=3.903$ 
The dummy row O3 is allotted a supply $=\sum_{\mathbf{j}=1}^{4} \psi_{\mathbf{j}}^{-1}(\beta)-\mathrm{R}^{*}=78.315-70.903=7.412$.

Hence the transformed new transportation problem with limited flow 70.903 is formulated and provided in table 5.3 .

Table 5.3: Transportation Problem with Impaired Flow

\begin{tabular}{|c|c|c|c|c|c|c|}
\hline $\mathbf{j}$ & $\mathbf{S} 1$ & $\mathbf{S} 2$ & $\mathbf{D 1}$ & $\mathbf{D} 2$ & $\mathbf{O 3}$ & $\phi_{\mathbf{i}}^{-1}(1-\alpha)$ \\
\hline $\mathrm{S} 1$ & 0 & 2.211 & 7.423 & 4.817 & 0 & 21.24 \\
\hline $\mathrm{S} 2$ & 2.211 & 0 & 3.211 & 6.423 & 0 & 22.24 \\
\hline $\mathrm{D} 1$ & 7.423 & 3.211 & 0 & 3.211 & 0 & 15.663 \\
\hline $\mathrm{D} 2$ & 4.817 & 6.423 & 3.211 & 0 & 0 & 15.663 \\
\hline $\mathrm{O} 3$ & 0 & 0 & 0 & 0 & $\mathrm{M}$ & 7.412 \\
\hline$\psi_{\mathbf{j}}^{-1}(\beta)$ & 15.663 & 15.663 & 20.48 & 26.509 & 3.903 & 82.218 \\
\hline
\end{tabular}

Using (P5) we have

$\min Z^{*}$

subject to

$$
\begin{gathered}
\sum_{i=1}^{4} \sum_{j=1}^{4}\left(\mu_{i j}+\frac{\sigma_{i j} \sqrt{3}}{\pi} \ln \frac{\alpha}{1-\alpha}\right) y_{i j}+M y_{55}-Z^{*} \leq 0 \\
\sum_{j=1}^{2} y_{i j}-\left(\mu_{i}+\frac{\sigma_{\mathbf{i}} \sqrt{3}}{\pi} \ln \frac{1-\alpha_{\mathbf{i}}}{\alpha_{\mathbf{i}}}\right)=15.663 \quad \forall i=1,2 \\
\sum_{j=1}^{5} y_{i j}+\left(\mu_{i}+\frac{\sigma_{\mathbf{i}} \sqrt{3}}{\pi} y_{i j}=15.663 \quad \forall i=3,4\right. \\
\sum_{i=1}^{5} y_{i j}-\left(\mu_{\mathbf{j}}+\frac{\sigma_{\mathbf{j}} \sqrt{3}}{\pi} \ln \frac{\beta_{\mathbf{j}}}{1-\beta_{\mathbf{j}}}\right)=15.663 \quad j=3,4 \\
\sum_{i=1}^{5} y_{i j}=15.663 \forall j=1,2 \\
\left.\mu_{\mathbf{j}}+\frac{\sigma_{\mathbf{j}} \sqrt{3}}{\pi} \ln \frac{\beta_{\mathbf{j}}}{1-\beta_{\mathbf{j}}}\right)-\sum_{i=1}^{5} y_{i j}=70.903 \quad j=5
\end{gathered}
$$

As the diagonal cell has unit cost zero we can ignore the allocations in them and we obtained the optimal basic feasible solution for the above transshipment problem with restricted flow is $\mathrm{Z}^{*}=32.009$. The optimal allocations are $y_{14}=3.434 ; y_{15}=2.143 ; y_{23}=4.817 ; y_{25}=1.76, y_{54}=7.412$. The original demand of D1=4.817 and D2=10.846 are satisfied.

\subsection{Example for Uncertain Transshipment Problem with Enhanced Flow}

Consider the same illustrative example discussed in section 5.1.

Let $\mathrm{R}^{*}$ be the total flow enhanced to 82.218. By taking the predetermined confidence level as $\alpha=0.9, \alpha_{\mathbf{i}}=0.9(\mathrm{i}=1,2,3,4)$ and $\beta_{\mathbf{j}}=0.9(\mathrm{j}=1,2,3,4)$ table 5.2 is formulated as table 5.4.

We introduce a dummy row and dummy column with each cell having a cost defined in P6 except $\mathrm{C}_{55}$ having cost M. The dummy row O3 is allotted a demand $=\mathrm{R}^{*}-\sum_{\mathrm{i}=1}^{4} \phi_{\mathrm{i}}^{-1}(1-\alpha)=82.218-74.806=7.412$. The dummy column O3 is 
allotted a supply $=\mathrm{R}^{*}-\sum_{\mathbf{j}=1}^{4} \psi_{\mathbf{j}}^{-1}(\beta)=82.218-78.315=3.903$

Table 5.4: Transportation Problem With Enhanced Flow

\begin{tabular}{|c|c|c|c|c|c|c|}
\hline $\mathbf{j}$ & $\mathbf{S 1}$ & $\mathbf{S 2}$ & $\mathbf{D 1}$ & $\mathbf{D 2}$ & $\mathbf{O 3}$ & $\phi_{\mathbf{i}}^{-1}(1-\alpha)$ \\
\hline $\mathrm{S} 1$ & 0 & 2.211 & 7.423 & 4.817 & 4.817 & 21.24 \\
\hline $\mathrm{S} 2$ & 2.211 & 0 & 3.211 & 6.423 & 3.211 & 22.24 \\
\hline $\mathrm{D} 1$ & 7.423 & 3.211 & 0 & 3.211 & 3.211 & 15.663 \\
\hline $\mathrm{D} 2$ & 4.817 & 6.423 & 3.211 & 0 & 4.817 & 15.663 \\
\hline $\mathrm{O} 3$ & 4.817 & 3.211 & 3.211 & 4.817 & $\mathrm{M}$ & 7.412 \\
\hline$\psi_{\mathbf{j}}^{-1}(\beta)$ & 15.663 & 15.663 & 20.48 & 26.509 & 3.903 & 82.218 \\
\hline
\end{tabular}

Since $\sum_{\mathrm{j}=3}^{4} \psi_{\mathbf{j}}^{-1}(\beta)>\sum_{\mathbf{i}=1}^{2} \phi_{\mathbf{i}}^{-1}(1-\alpha)$ we have $\mathrm{T}=\operatorname{Max}\left(\sum_{\mathrm{j}=3}^{4} \psi_{\mathbf{j}}^{-1}(\beta)>\sum_{\mathbf{i}=1}^{2} \phi_{\mathbf{i}}^{-1}(1-\alpha)\right)=15.663$ and convert the problem to balanced Transshipment problem and given table 5.2. Let $\mathrm{R}^{*}$ be the total flow restricted to 82.218.

We introduce a dummy row and dummy column with each cell having a cost 0 except $\mathrm{C}_{55}$ having cost $\mathrm{M}$.

The dummy row $\mathrm{O} 3$ is allotted a demand $=\mathrm{R}^{*}-\sum_{\mathrm{i}=1}^{4} \phi_{\mathrm{i}}^{-1}(1-\alpha)=82.218-74.806=7.412$

The dummy column O3 is allotted a supply $=\mathrm{R}^{*}-\sum_{\mathbf{j}=1}^{4} \psi_{\mathbf{j}}^{-1}(\beta)=82.218-78.315=3.903$.

Using (P7) we have

$\min Z^{*}$

subject to

$$
\begin{aligned}
& \sum_{i=1}^{4} \sum_{j=1}^{4}\left(\mu_{i j}+\frac{\sigma_{i j} \sqrt{3}}{\pi} \ln \frac{\alpha}{1-\alpha}\right) y_{i j}+4.817 y_{15}+3.211 y_{25}+3.211 y_{35}+ \\
& 4.817 y_{45}+4.817 y_{51}+3.211 y_{52}+3.211 y_{53}+4.817 y_{54}+M y_{55}-Z^{*} \leq 0 \\
& \sum_{j=1}^{2} y_{i j}-\left(\mu_{i}+\frac{\sigma_{\mathbf{i}} \sqrt{3}}{\pi} \ln \frac{1-\alpha_{\mathbf{i}}}{\alpha_{\mathbf{i}}}\right)=15.663 \quad \forall i=1,2 \\
& \sum_{j=1}^{5} y_{i j}+\left(\mu_{i}+\frac{\sigma_{i} \sqrt{3}}{\pi} \ln \frac{1-\alpha_{\mathbf{i}}}{\alpha_{\mathbf{i}}}\right)=70.903 i=5 \\
& \sum_{i=1}^{5} y_{i j}-\left(\mu_{\mathbf{j}}+\frac{\sigma_{\mathbf{j}} \sqrt{3}}{\pi} \ln \frac{\beta_{\mathbf{j}}}{1-\beta_{\mathbf{j}}}\right)=15.663 \quad \forall i=3,4 \\
& \left(\sum_{i=1}^{5} y_{i j}=15.663 \forall j=1,2\right. \\
& \left.\mu_{\mathbf{j}}+\frac{\sigma_{\mathbf{j}} \sqrt{3}}{\pi} \ln \frac{\beta_{\mathbf{j}}}{1-\beta_{\mathbf{j}}}\right)-\sum_{i=1}^{5} y_{i j}=70.903 \quad j=5
\end{aligned}
$$

As the diagonal cell has unit cost zero we can ignore the allocations in them and we obtained the optimal basic feasible solution for the above transshipment problem with restricted flow is $\mathrm{Z}^{*}=80.245$. The optimal allocations are 
$y_{14}=5.577 ; y_{23}=2.674 ; y_{25}=3.903 ; y_{43}=2.143 ; y_{54}=5.269$. The original demand of D1=4.817 and D2=10.846 are satisfied.

\section{CONCLUSIONS}

In this paper, variants of uncertain transshipment problem are discussed which may arise due to various emergency situations. We have to either restrict or enhance the transshipment flow based on the market scenario which may help the decision maker in any crisis. An uncertain transshipment problem with impaired and enhanced flow is considered and a solution procedure is explained to obtain the optimal solution. The uncertain transshipment problem is transformed to an equivalent deterministic model by using chance constraint model and then the optimal solution is reached. Illustrative examples are provided to understand the methodology developed in this paper.

\section{REFERENCES}

1. Alex Orden, The Transshipment Problem, Management Science 2(3):276-285, 1956.

2. Verner G. Hurt and Thomas E,. Tramel, Alternative Formulations of the transshipment problems, Journal of Farm Economics, 47(3):763-773,1965.

3. Garg, R. \& Prakash, S. (1985). Time minimizing transshipment problem.Indian J. Pure Appl. Math. 16(5):449-460.

4. Herer, Y. T., \& Tzur, M. (2001). The Dynamic Transshipment Problem, Naval Research Logistics, Vol. 48: 386-408.

5. KhuranaA, \& Arora SR (2011). Solving transshipment problems with mixed constraints. Int. J. Manag. Sci. Eng. Manag. $6(4): 292-297$

6. Kanpuri, Prafull, and Abhishekh Kumar. "A Study of Inventory on Supply Chain Management through Lateral Transshipment with Special Reference of ABC Company." International Journal of Mechanical and Production Engineering Research and Development (IJMPERD) 4.2 (2014): 21-34.

7. Khurana, A. (2015). Variants of transshipment problem. European Transport Research Review 7: 11 DOI 10.1007/s12544-0150154-8.

8. Khurana, A. (2015b). On a class of three-dimensional transshipment problems, Int. J. Shipping and transport Logistics, Vol. 7 , No. 4, pp. 407-425.

9. Khurana, A., Verma, T. \& Arora S. R. (2015). Solving time minimizing transshipment problem. Int. J. Shipping and transport Logistics, Vol. 7, No. 2, pp. 137-155.

10. Ahmed, A. Kaleel, C. B. Senthilkumar, and S. Nallusamy. "Study on environmental impact through analysis of big data for sustainable and green supply chain management." Int. J. Mech. Prod. Eng. Res. Dev. 8 (2018): 1245-1254.

11. Gregory Okiemuteagadaga, Nsikanpaulakpan, Transshipment Problem and Its Variants: A Review,Mathematical Theory and Modeling, Vol.7, No.2, 2017

12. NagoorGani, A., Baskaran, R. \& Mohamed Assarudeen, S. N. (2012). Mixed Constraint Fuzzy Transshipment Problem.Applied Mathematical Sciences, Vol. 6, No. 48: 2385-2394.

13. Rajendran, P., \& Pandian, P. (2012). Solving Fully Interval Transshipment Problems. International Mathematical Forum, Vol. 7, No. 41: $2027-2035$.

14. Hmiden, M., Ben Said, L. \& Ghedira, K. (2009). Transshipment problem with uncertain customer demands and transfer lead time. Proceedings of the International Conference on Computer \& Industrial Engineering, University of Technology of Troyes, 
France 6-8 July 2009.

15. Liu, B, Uncertainty Theory, 2nd edition, Springer-Verlag, Berlin, 2007.

16. Liu, B, Uncertainty Theory, 5th edition, Springer-Verlag, Berlin, 2018.

17. Yuhong Sheng, Kai Yao,A Transportation Model with UncertainCosts and Demands,

18. International journal on information 15(8), August 2012.

19. Shukla, Kunal Tarunkumar. "Fuzzy Floyd's algorithm to find shortest route between nodes under uncertain environment." Int $J$ Math ComputAppl Res 3.5 (2013): 34-54.

20. Bo Zhang, Jin Peng, Shengguo Li, Lin Chen, (2016), Fixed charge solid transportation problem in uncertain environment and its algorithm, Computers and Industrial Engineering, 102, 186-197.

21. Subhakanta Dash and S. P. Mohanty,(2016)Transportation Problem with Uncertain Cost, Rough Demand and Supply, International Journal of Operations Research Vol 13, No.3, 085-094.

22. Yufu Ning,Hua Ke and Zongfei Fu, (2015), Methodologies and application,Triangular entropy of uncertain variables with application to portfolio selection, Soft Computer, 19:2203-2209,DOI 10.1007/s00500-014-1402-x.

23. Liu and Chen(2015), Uncertain Multiobjective Programming and Uncertain Goal Programming, Journal of uncertainty analysis and applications, doi10.1186/s40467-015-00366.

24. Salem, ISLAM I., and KHALED G. El-Sakty. "Port selection criteria and its impact on port competitiveness." Int J Human SocSci 3.6 (2014): 29-36.

25. DeyiMou and Xiaoding Chang(2014),An Uncertain Programming for the Integrated Planning ofProduction and Transportation, Mathematical Problems in Engineering, Volume 2014,http://dx.doi.org/10.1155/2014/419358.

26. Liu, B. (2009a). Some research problems in uncertainty theory, Journal of UncertainSystems, 3(1), 3-10.

27. Liu B,(2010), Uncertainty Theory: A Branch of Mathematics for Modeling Human Uncertainty, Springer-Verlag, Berlin.

28. Liu B, and Yao K(2012), Uncertain integral with respect to multiple canonical processes,Journal of Uncertain Systems, Vol.6, No.4, 250-255. 